STRUCTURAL SCIENCE CRYSTAL ENGINEERING MATERIALS

ISSN 2052-5206

\section{Crystal Structures. Patterns and Symmetry. By Michael O'Keeffe and Bruce G. Hyde. Dover, 2020. Paperback, pp. xvi+461. Price USD 39.95. ISBN 978-0- 486-83654-6}

\author{
Massimo Nespolo*
}

Université de Lorraine, CNRS, CRM2, Nancy, France. *Correspondence e-mail: massimo.nespolo@univ-lorraine.fr

Dover publications continues its commendable task of republishing classical textbooks that are out of print, often hard to get even in the second-hand market. This year they republished one of the books that has ignited the now extremely flourishing field of reticular chemistry: Crystal Structures. Patterns and Symmetry, by O'Keeffe and Hyde. Usually, a re-publication is briefly announced in our book received section. We make an exception in this case because of the importance of the book, because it originally appeared in a minor series (Monographs of the Mineralogical Society of America), and because it has long been out of print. With respect to the original publication, dating back to 1996, the original Foreward has been omitted and a chapter 'Notes', by Michael O'Keeffe and Davide Proserpio, has been added.

The book is composed of seven chapters and five appendices; it can be divided into two large sections: one about crystallographic symmetry (chapters 1 to 4 ), the other about the description of crystal structures in terms of polyhedra and nets (chapters 5 to 7). It ends with five appendices, a list of reference books, the already mentioned notes to the Dover edition, and an extensive index, which includes a formula index. Each chapter ends with 'Notes', meant to dwell somehow deeper (often these are among the most interesting parts of the book) and a set of exercises (without solution).

Chapter 1 (27 pages) presents Symmetry in two dimensions. Some statements are today somewhat outdated, such as the use of shortened symbols for space groups ( $\mathrm{pmm}$ and $\mathrm{p} 4 \mathrm{~g}$ for $p 2 \mathrm{~mm}$ and $p 4 g m$, respectively, have not been used for a long time). Some terms are used before being defined (symmetry element, crystal system, conventional cell) and some are imprecise: a crystal class is not a point group but related to it; the difference between a group and a type of group is not made. In many cases the difference is understood from the context, yet the lack of explicit differentiation sometimes leads to astonishing statements. One example occurs on page 82 , where we read 'the space group remains the same so this is an example of ordering producing an isomorphic subgroup'. Obviously, the space group cannot be the same (but of the same type) if one is the subgroup of the other! The same problem occurs when we read (page 87) that ' $P 3_{1} 21$ is seen to be an isomorphic subgroup of index 2 of $P 3_{2} 21$ ': without specifying the transformation matrices these are just types of space group and the statement is meaningless. Despite these venial sins and the prudent and too humble statement (page 1) 'No attempt is made to be either rigorous or complete', this book is far more rigorous than what we can find in a number of more recent books, whose authors shamelessly write about something they do not understand (examples can be found among the book reviews I have published in the last few years).

Chapter 2 (30 pages) introduces Three-dimensional point groups. It consists of a systematic analysis of the crystallographic (types) of point groups through 3D drawings and stereographic projections, with a brief analysis of the geometric restrictions. The term 'dihedral groups' is used to indicate pure rotation groups of type $\mathrm{N} 2(2)$, i.e. 222, 32, 422, 622 , at odds with the definition in mathematics according to which a dihedral group is the group of symmetries of a regular polygon and as such includes rotations as well as reflections. Deformed tetrahedra are still called tetrahedra instead of disphenoids, although the term does show up (page 142) in between sphenoids and bisdisphenoids. I found rather disturbing the imprecise use of the term 'system' (pages 45-46), where we 
read that 'in the orthorhombic system there are three mutually twofold perpendicular axes'. A 'system' is a class (category used for classification); the statement above applies to the groups classified in that system, not to the system itself.

Chapter 3 (41 pages) is devoted to Three-dimensional space groups and starts with a short introduction to (Bravais) lattices, which are described in more detail in the following chapter. One should regret the absence of the concept of crystal family, which unavoidably leads to awkward expressions like 'hexagonal (sensu lato)' and 'hexagonal (sensu strictu)'. Nevertheless, the text is exempt from the typical confusion between trigonal and rhombohedral, which pollutes so many books. In the list of types of glide planes, $e$ and $g$ are missing. The treatment is rigorous and pedagogical at the same time, and spans up to the extended Hermann-Mauguin symbols, seldom if ever seen in textbooks, despite their paramount importance for understanding how space-group symbols are constructed. The statement (page 73) 'in the class $\overline{4} 2 m$ there are two distinct space groups, $P \overline{4} m 2$ and $P \overline{4} 2 m$ ' is somewhat unfortunate in that it could be interpreted as if there were only two (types of) space groups in this (geometric) crystal class. The exceptional case of $I 23$ versus $I 2_{1} 3$ (page 76) could have been introduced through the respective subgroups I222 versus $I 2_{1} 2_{1} 2_{1}$. The footnote on page 83 is quite disturbing: 'Only crystallographers really believe in fractional atoms'. I wonder if the authors really do consider that we crystallographers have such a belief! The notes to this chapter briefly introduce the matrix representation of symmetry operations, alternative choices of unit cells and origin, equivalent descriptions of crystal structures (but without mentioning normalizers) as well as the notion of lattice complex. Given the importance of this concept in the second part of the book, it would have deserved a place in the body of the chapter, introduced by the 'parent' concepts of crystallographic orbit (or point configuration, depending on the school you come from).

Chapter 4 (33 pages) has the title Lattice geometry and is devoted to simple crystallographic calculations, where, contrary to the $I U C r$ standards, the unfortunate choice of representing both covariant and contravariant quantities as column matrices is made. Unfortunately, several misunderstandings quite common in the literature appear here as well. The statement according to which 'A direction in a crystal is specified by three integers' is valid only if a primitive basis is used (Nespolo, 2017) and is contradicted in the footnote on page 111; the use of Weber indices (whose name does not appear anywhere in the book), far from having 'advantages', is a source of confusion and misunderstandings (Nespolo, 2018). Miller indices are 'integers with no factor common to all three' only if a primitive basis is used (Nespolo, 2015). Several examples are worked out in detail, but a more extensive use of the metric tensor would have made them easier.

Chapter 5 ( 76 pages) opens the second part of the book by discussing Polyhedra and tilings. It is a fascinating and approachable journey through regular, semi-regular, truncated, capped polyhedra, clusters of polyhedra, and through the crystal structure types where these polyhedra occur, more or less deformed, as coordination polyhedra. It is followed by an analysis of circle packing and tilings of the plane, as a basis for the symmetry of layers. The authors correctly use subperiodic groups (layer groups, rod groups) to describe the structures of diperiodic and monoperiodic building blocks. Subperiodic groups have been known for decades but are seldom described in textbooks dealing with crystal structures and many authors, even today, seem unaware of their existence. Instead, they incorrectly use two-dimensional or monodimensional groups, leading to absurd yet widespread terms like 'low-dimensional structures' (Nespolo, 2019). The chapter ends with a short excursion into aperiodic tilings and quasicrystals, followed by the Notes with some notions about Schlegel diagrams, adjacency matrices and the construction of polyhedra.

Chapter 6 (81 pages) is devoted to Sphere and cylinder packings. More than half of the chapter deals with sphere packings, describing not only how various polytypes can be obtained by stacking equivalent layers of spheres, but also the possible patterns of filling the cavities that arise from this stacking. The symbols chosen to describe the spheres and the cavities are not the most general ones. Zoltai \& Stout (1984) introduced a more general notation, which unfortunately is less well known (we have tried to draw more attention to it recently: Umayahara \& Nespolo, 2018). Zoltai \& Stout had also considered the fact that The term 'close packing' is sometimes used rather loosely (page 271) by introducing the term symmetric packing; it is regrettable that their work is so frequently overlooked. Particularly appreciated is the care with which the difference between the structure obtained by stacking of layers of spheres and its lattice is emphasized, something that too often is misunderstood even in highranking publications by highly respected authors. Something more could have written about the 'description of paths between packings [...] that preserve as much symmetry as possible' (page 228), by specifying that these paths are typically realized in displacive phase transformations, whereas they are of much less relevance for reconstructive phase transformations (Müller, 2017). The unit-cell level transformation of different types of structure is described in detail but incorrectly called 'twinning' (page 231), instead of 'cell-twinning' (Takéuchi, 1997), a term which is still insufficiently known among structure scientists. The authors have been careful to provide many details to anchor their geometric description to the physical properties of the structures, as in case of auxetic materials (materials with negative Poisson's ratio: page 242) although this term, possibly less known at the time of the first printing, is not used, or of high-temperature superconductors (page 261), although today's 'high-temperature' is significantly different from what was considered 25 years ago. The section on cylinder packings presents structures built by rods and is a rare example of application of rod groups, which seem frequently unknown among structural scientists. The chapter ends with a detailed analysis of sphere packings in terms of lattice complexes: this collection of examples should convince even the most sceptical structural scientist that lattice complexes are a useful tool, not just an 
abstract concept limited to the 'esoteric' world of mathematical crystallography.

Chapter 7 (92 pages), devoted to Nest and infinite polyhedra, represents the culmination of this journey in the kingdom of structural symmetry. Whereas the previous chapter was centred around polyhedra with high coordination numbers, this last chapter deals with arrangement of points which correspond to low coordination, 3- and 4-connected nets. The importance of these nets was already evident at the time of the first printing of the book (e.g. in the study of zeolites) and has since further increased, with the impressive developments of metal-organic frameworks (MOFs). In this chapter, nets are analysed with a minimal use of graph theory; the focus is on zeolites, feldspars and nets that occur as components of more complex structures, such as diamond and quartz. The survey is extensive and detailed.

The book ends with five appendices. The first appendix is about subperiodic groups (a topic insufficiently known about by structural scientists, not only at the time of the first publication) and Curie groups (not referred to as such by the authors): groups containing at least one axis of infinite order, by which we represent the symmetry of physical properties of crystals). The second appendix gives some glimpses into higher-dimensional space, whereas the third presents basic notions about topology of nets and introduces minimal surfaces, which allow us to follow paths inside a crystal structure (possible connections with percolation theory are evident but outside the topic of the book). The fourth appendix introduces large polyhedra and sounds likes a premonition of the development of MOFs (remember, the book was first published in 1996). The last appendix presents data on the crystal structures described in the various chapters and a summary of symbols of symmetry groups.

Overall, despite being almost 25 years old, Crystal Structures. Patterns and Symmetry relevant, even if some topics have since been significantly developed. Its republication is therefore a most welcome initiative, although we would have preferred a second edition with some updates and corrections. At least, a list of errata could have been added. For the readers' interest, here are the few incorrect expressions that I have noted:

page 29: identity is a symmetry operation, not element; page 49: $\overline{3}^{4}=\overline{1}$ instead of $\overline{3}^{3}=\overline{1}$;

page 51 (last line): parentheses used instead of brackets to indicate lattice directions;

page 68: 'primitive cell translations' should read 'centred cell translations';

the statement (page 81) 'any kind of subgroup is possible for space groups with centred lattices' (meaning either translationengleiche and klassengleiche) is really unfortunate, the restriction of centred lattices being totally unnecessary; page 86: 'a crystal [...] composed of two parts' is not 'composed of twins' but is a twin;

software cited in the book (e.g. EUTAX) are outdated and no longer available: a list of up-to-date alternatives would have been useful.

A few typos have carried over to the new printing, but these do not hinder the understanding. Occasionally, non-standard notations are used. Antisymmetry is indicated by underline (page 54) instead than a prime, as almost universally agreed. A curiosity worth mentioning is the first footnote on page 32 , where the authors mention that the use of ' 3 bar' or 'bar 3' for $\overline{3}$ seems to be equally spread among 'the fraction of the world population that cares'. According to Mike Glazer (personal communication), ' 3 bar', preferred by these authors, is the American version, while 'bar 3' is the British one.

This book was published as Volume I. The second volume, which should have been even more extensive than the first, was never published. One reason is technical: the material that had been prepared by the authors became obsolete due to the rapid evolution of the software (text and graphical editing, and especially crystal structure representation). But more importantly, crystal chemistry was changing dramatically, and structures constructed with organic ligands took such a predominant role that it led to switching the main target of the authors' research towards what today is known as reticular chemistry. The Notes to the Dover edition that end this new printing is meant to be a pointer to the evolution after 'Volume I' was printed. It calls attention to several topics that, to the best of my knowledge, are missing from most, possibly, all, textbooks: (a) crystallographic descriptions of knots, links and weavings, all hot topics in current crystal chemistry; $(b)$ invariant periodic line sets (especially important in the description of 3-periodic weaving); (c) the modern theory of periodic tilings and its relevance to enumeration and description of nets. The Notes are an important addition that for sure widens the already large audience of interested readers.

\section{References}

Müller, U. (2017). Symmetry Relationships between Crystal Structures Applications of Crystallographic Group Theory in Crystal Chemistry. Oxford: Oxford University Press.

Nespolo, M. (2015). J. Appl. Cryst. 48, 1290-1298.

Nespolo, M. (2017). J. Appl. Cryst. 50, 1541-1544.

Nespolo, M. (2018). J. Appl. Cryst. 51, 1221-1225.

Nespolo, M. (2019). J. Appl. Cryst. 52, 451-456.

Takéuchi, Y. (1997). Tropochemical Cell-Twinning. A StructureBuilding Mechanism in Crystalline Solids. Tokyo: Terra Scientific Publishing Company.

Umayahara, A. \& Nespolo, M. (2018). Z. Kristallogr. 233, 179-203.

Zoltai, T. \& Stout, J. H. (1984). Mineralogy. Concepts and Principles. Minneapolis: Burgess. 